\title{
Judicious use of personal protective equipment to prevent the spread of COVID-19 in maternity units
}

More than 300 women give birth in South Africa (SA) every day. ${ }^{[1]}$ An increasing number of midwives, obstetricians and gynaecologists have tested positive for COVID-19 in SA, and this has led to the closures of maternity units. Recently, General Justice Gizenga Mpanza Regional Hospital in KwaZulu-Natal Province in SA closed after 16 healthcare workers (HCWs) and patients tested positive for SARS-CoV-2 virus, necessitating the redirection of patients to other healthcare centres in the area. ${ }^{[2]}$ Antenatal care and safe childbirth are crucial and cannot be postponed, making pregnant women and obstetric HCWs a vulnerable population for healthcare facility transmission. Reducing antenatal care consultations is associated with increased maternal and perinatal morbidity and mortality. ${ }^{[3]}$ Therefore it is imperative that maternity services remain functional.

The national guidelines for COVID-19 in pregnancy stipulate that only individuals with symptoms should be tested for the SARS-CoV-2 virus. ${ }^{[4]}$ According to a recent study in New York, $13 \%$ of pregnant women who were asymptomatic tested positive for COVID-19 at delivery, and it is estimated that the prevalence of asymptomatic carriers in labour can be as high as $30 \% \cdot{ }^{[5]}$ Universal testing is currently not achievable, ${ }^{[3]}$ therefore all patients presenting for maternity care should be regarded as asymptomatic carriers. The current recommendations for infection prevention and control (IPC) include: (i) the use of surgical masks for both patients and HCWs; (ii) wearing an apron, gloves and eye protection; and (iii) minimising time spent exposed to patients. In the outpatient clinics and inpatient antenatal and postnatal ward settings, personal protective equipment (PPE) can be worn for the entire session without changing between each patient, as long as it is safe to do so. In addition, regular handwashing and social distancing must be adhered to. ${ }^{[4]}$

Aerosol-generating procedures (AGPs) require a higher level of PPE (N95 respirators or equivalent) for patients under investigation (PUIs) and confirmed SARS-CoV-2/COVID-19- positive patients. ${ }^{[3]}$ Caesarean section under general anaesthesia is considered an AGP. ${ }^{[4,6]}$ Labour and delivery present a unique situation because women use all their energy and strength, especially during the second stage of labour. It is not uncommon for women in labour to scream and exhale expulsively, and both can arguably be considered AGPs. Furthermore, vomiting, expulsion of liquor and faecal contamination often accompany the delivery process. ${ }^{[3,6]}$ There are conflicting views on whether a normal vaginal delivery is an AGP requiring additional PPE, or a dropletproducing procedure wherein standard PPE is adequate..$^{[4,6,7]}$ The prevalence of asymptomatic women presenting in labour in SA remains unknown. While the patient is expected to wear a surgical mask at all times, this is not always achievable during all deliveries owing to the increased energy demands associated with pushing. In order to reduce potential exposure, the presence of companions such as doulas, partners or other family members has also been restricted, posing an additional ethical dilemma.

It remains a priority to maintain the health and safety of our HCWs to ensure continuity of quality care. There is evidence that appropriate use of PPE reduces disease transmission and protects staff. ${ }^{[8]}$ It is therefore important that PPE is used appropriately and not wastefully.

Our recommendations for infection prevention control in maternity units in SA are as follows:

- Adequate PPE should be available for HCWs, and surgical masks for patients.

- HCWs should be trained on donning and doffing of PPE.

- PPE standards should be integrated into healthcare facility inspections.

- All patients should be managed as PUIs during labour and delivery, i.e. HCWs to use N95 respirators (or equivalent), or surgical face masks together with face shields.

- Movement of patients should be minimised between areas in the labour ward.

- There should be minimum change of staff per patient.

- If possible, surgical drapes or shields should be utilised between patient and HCW as part of routine PPE during labour and delivery. ${ }^{[6,9]}$

- There should be adherence to national guidelines relating to self-isolation, and strict guidelines for testing of staff, shutting down facilities and universal testing.

Implementing stricter IPC protocols will protect HCWs and pregnant women. Despite the challenges posed by the pandemic, childbirth is a memorable experience. It remains our responsibility to create a safe environment for pregnant women and HCWs, and to promote the judicious use of PPE.

Sumaiya Adam, MB ChB, FCOG (SA), MMed (O\&G), Cert Maternal Fetal Med (SA), PGCHE, PhD

Department of Obstetrics and Gynaecology, School of Medicine, Faculty of Health Sciences, University of Pretoria, South Africa sumaiya.adam@up.ac.za

Salome Maswime, MB BCh, FCOG (SA), MMed (O\&G), PhD Global Surgery Division, Department of Surgery, Faculty of Health Sciences, University of Cape Town, South Africa salome.maswime@uct.ac.za

Priya Soma-Pillay, MB ChB, Dip Obs (SA), FCOG (SA), MMed (O\&G), Cert Maternal Fetal Med (SA), PhD (O\&G) Department of Obstetrics and Gynaecology, School of Medicine, Faculty of Health Sciences, University of Pretoria, South Africa priya.somapillay@up.ac.za

Mushi Matjila, BSc (Biochem Microbiol), MB ChB, Dip Obs (SA), FCOG (SA), PhD

Department of Obstetrics and Gynaecology, Faculty of Health Sciences, University of Cape Town, Cape Town, South Africa mushi.matjila@uct.ac.za 
Lawrence Chauke, MB ChB, FCOG (SA), MMed (O\&G), Dip HIV (SA) Department of Obstetrics and Gynaecology, Faculty of Health Sciences, University of the Witwatersrand, Johannesburg, South Africa lawrence.chauke@wits.ac.za

Matthys H Botha, MB ChB, FCOG (SA), MMed (O\&G), PhD Department of Obstetrics and Gynaecology, Faculty of Health Sciences, Stellenbosch University and Tygerberg Hospital, Cape Town, South Africa

mhbotha@sun.ac.za

Robert Pattinson, BSc, MB ChB, MMed, FCOG, FRCOG, MD SAMRC/UP Maternal and Infant Health Care Strategies Unit, Pretoria, South Africa robert.pattinson@up.ac.za

S Afr J Obstet Gynaecol 2020;26(1):2-3. https://doi.org/10.7196/SAJOG.2020.v26i1.1605
1. Macrotrends. South Africa Birth Rate 1950 - 2020. https://www.macrotrends.net/countries/ZAF/ south-africa/birth-rate (accessed 13 July 2020)

2. KwaZulu-Natal Department of Health. Gen. Justice Gizenga Mpanza Regional Hospital to temporarily stop taking patients, following lab-confirmed COVID-19 infections; priority now on protection of other patients and staff, and to allow for the decontamination of the hospital. Pietermaritzburg: KZN DoH, 2020. http://www.kznhealth.gov.za/mediarelease/2020/gjg-mpanza-regional-hospital-totemporarily-stop-taking-patients-04052020.htm (accessed 13 July 2020).

3. Boelig RC, Manuck T, Oliver EA, et al. Labor and delivery guidance for COVID-19. AJOG 2020;2(2):Suppl. https://doi.org/10.1016/j.ajogmf.2020.100110

4. Pattinson RC, ed. Framework and guidelines for maternal and neonatal care during a crisis. COVID19 response. Pretoria: National Department of Health, 2020. https://www.hst.org.za/Covid19/ Documents/COVID-19\%20Maternal\%20\%20newborn\%20care\%20guidelines\%202020-04-30\%20 -\%20revised.pdf (accessed 13 July 2020).

5. Sutton D, Fuchs K, D’Alton M, Goffman D. Universal screening for SARS-CoV-2 in women admitted for delivery. N Engl J Med 2020;382:2163-2164. https://doi.org/10.1056/NEJMc2009316

6. ObG Project. COVID-19, PPE and second stage of labor: Current US guidance. ObG Project, 2020. https://www.obgproject.com/2020/03/31/COVID-19-ppe-and-second-stage-of-labor-current-usguidance/ (accessed 13 July 2020).

7. Public Health England, Department of Health and Social Care, NHS England. New personal protective equipment (PPE) guidance for NHS teams. https://www.gov.uk/government/news/newpersonal-protective-equipment-ppe-guidance-for-nhs-teams (accessed 13 July 2020).

8. Cook T. Personal protective equipment during the COVID-19 pandemic - a narrative review Anaesthesia 2020 (epub 4 April 2020). https://doi.org/10.1111/anae.15071

9. Sahin D, Erol SA, Tanacan A, Ozcan N, Keskin HL, Tekin OM. Protective equipment to use in the vaginal delivery of the pregnant woman with suspected or diagnosed coronavirus disease 2019: Delivery table shield. AJOG 2020 (epub 14 June 2020). https://doi.org/10.1016/j.ajog.2020.06.021 